\title{
Effects of arsenic and moisture regime on nutrient content and their uptake by NERICA rice
}

\author{
S. Bhattacherjee ${ }^{1}$, M. A. H. Chowdhury ${ }^{1^{*}}$, M. H. Rahman ${ }^{2}$ and B. K. Saha ${ }^{1}$ \\ ${ }^{1}$ Department of Agricultural Chemistry, Bangladesh Agricultural University, Mymensingh-2202 and ${ }^{2}$ Soil Science \\ Division, Bangladesh Institute of Nuclear Agriculture, Mymensingh, Bangladesh, *E-mail: akhterbau11@gmail.com
}

\begin{abstract}
A pot experiment was carried out in the net house of Bangladesh Institute of Nuclear Agriculture (BINA) to study nutrient content and their uptake by different rice varieties (NERICA-1, NERICA-10 and BRRI dhan48) in response to different levels of arsenic (As) under varying moisture regimes. Three levels of As were added viz. 0, 30, 60 ppm to the pot soil from sodium arsenate $\left(\mathrm{Na}_{2} \mathrm{HAsO}_{4} .7 \mathrm{H}_{2} \mathrm{O}\right)$. After establishment of crops the moisture level of soil was adjusted to maintain flooded, $50 \%$ and $100 \%$ field capacity condition during crop growing period. The experiment was laid out in a Completely Randomized Design (CRD), with three replications. The amount of N, P, K, S and Zn was calculated as per their rate of application and was applied to the individual pot. Higher levels of As adversely affected the nutrient content and their uptake by rice except $\mathrm{N}$ contents. Nitrogen content increased with the increase of As level. Higher amount of nutrient content and uptake was recorded in BRRI dhan48 and flood condition enhanced higher nutrient content as well as uptake by rice. This study suggests the possible management of moisture regime and considering less As susceptible variety, which might reduce the toxic effects of As on nutrient uptake.
\end{abstract}

Keywords: Arsenic level, Moisture regime, Rice varieties, Nutrient contents, Uptake

\section{Introduction}

Rice is the principal cereal crop in Bangladesh that plays the most important role in the national economy. Bangladesh is a delta of high As contamination in groundwater and the water being widely used for irrigation. Irrigation of agricultural land with As-contaminated water or groundwater, particularly in Bangladesh, India and South-east Asia, can easily be accumulated As in both soils and plants, causing risks to soil ecosystems and human health (Martin et al., 1992; Xie \& Huang, 1998; Meharg \& Rahman, 2003). Rice is more susceptible to arsenic toxicity compared to upland crops, because of an increase in both the bioavailability and toxicity of arsenic under the submerged soil of paddy fields ( Horswell and Speir, 2006). Rahman et al., (2008) investigated the severity of straight head in rice (cv. BRRI dhan29) which was increased significantly up to $50 \mathrm{mg}$ of $\mathrm{As} \mathrm{kg}^{-1}$ soil treatments. Straight head resulted in sterile florets with distorted lemma and palea, reduced plant height, tillering, panicle length and grain yield. Straight head caused approximately $17-100 \%$ sterile florets/spikelet formation and about $16-100 \%$ loss of

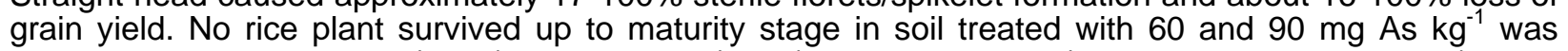
reported by Rahman et al., (2007). Wang et al., (2006) reported that As (roxarsone or arsanilic acid) could significantly reduce plant height, number of effective tiller, straw yield and grain yield. Arsenic significantly reduced Phosphorus concentration in rice (Zhu, 2003). Arsenic uptake and accumulation in rice plant from irrigation water may differ depending on cultivars used (Xie and Huang, 1998). Rice is the staple food in this country, any adverse effects on nutrient content of rice due to arsenic contaminated irrigation water would badly cause nutrient deficiency problem. This research attempted to reduce toxic effects of As on nutrient content in rice by using different variety and moisture regime.

\section{Materials and Methods}

A pot experiment was carried out during Aus season (May- August) of 2012 at the net house of Bangladesh Institute of Nuclear Agriculture (BINA), Mymensingh having low arsenic content $(2.1 \mathrm{mg} / \mathrm{kg})$ in soil. Before the set up of the experiment soil was tasted and found that silt loam in texture with $6.7 \mathrm{pH}$, $1.53 \%$ organic matter, $0.12 \%$ total $\mathrm{N}, 9.8 \mathrm{mg} / \mathrm{kg}$ available $\mathrm{P}, 10.5 \mathrm{mg} / \mathrm{kg}$ available $\mathrm{S}, 0.11 \mathrm{me} / 100 \mathrm{~g}$ exchangeable $\mathrm{K}$ and $38.81 \%$ field capacity. The size of each pot was $43 \mathrm{~cm}$ in diameter and $40 \mathrm{~cm}$ in height. There were 27 treatments consisting of three doses of As $(0,30$ and $60 \mathrm{ppm})$, three moisture regime (50\% field capacity, $100 \%$ field capacity and flood condition) and three rice varieties (NERICA-1, NERICA-10 and BRRI dhan48). The source of added As was sodium arsenate $\left(\mathrm{Na}_{2} \mathrm{HAsO}_{4} \cdot 7 \mathrm{H}_{2} \mathrm{O}\right)$. The experiment was conducted in a three factorial completely randomized design with three replications. The seeds were sown in the pots on May, 2012. Arsenic at concentrations $0 \mathrm{ppm}, 30 \mathrm{ppm}$ and $60 \mathrm{ppm}$ was mixed with the soils one week before sowing of seeds. To maintain flooded condition, $50 \%$ and $100 \%$ field capacity, required amount of water was added. Plastic pots were weighed daily and the difference in the weight of the pot was ascribed to water lost by evaporation. The lost water was replenished by adding an equal volume of water to the pot. Every pot had received $9 \mathrm{~g} \mathrm{~N}$ from urea, $4 \mathrm{~g} \mathrm{P}$ from TSP, $3.5 \mathrm{~g} \mathrm{~K}$ from muriate of potash (MoP) and $1.5 \mathrm{~g} \mathrm{~S}$ from gypsum. The full amount of all other fertilizers except nitrogen 
was applied as basal to the individual pot. Nitrogen was added in three splits, The first split of urea was applied at 15 days after seed sowing i.e. early vegetative stage, the second split of urea was applied at 40 days after seed sowing i.e. at maximum tillering stage and the third split was applied at 75 days after seed sowing i.e. at panicle initiation stage. Intercultural operations such as weeding and spraying insecticides were done whenever required. The crop was harvested at maturity and the data on yield and yield parameters were recorded. Plants from each pot were measured and averaged to record the yield contributing characters. Grain and straw samples were analyzed for N, P, K and S contents and uptake. All data were statistically analyzed following F-test and the difference between treatment means was adjudged by Duncan's Multiple Range Test.

\section{Results and Discussion}

Nitrogen content: Remarkable effect of different doses of added As was observed on the $\mathrm{N}$ content in grain and straw of rice (Table 1). The level of $\mathrm{N}$ content was higher when high amount of As was added. The highest $\mathrm{N}$ content $(0.515 \%$ in grain and $0.592 \%$ in straw) was found with $60 \mathrm{ppm}$ dose of added As. Straw N-content was lowest $(0.536 \%)$ with $0 \mathrm{ppm}$ As treatment and lowest grain N-content $(0.470 \%)$ was recorded at $30 \mathrm{ppm}$ As treatment. This finding agrees with Begum et al.; 2008 who reported that the $\mathrm{N}$ content in grain and straw increased with increasing addition of arsenic. Different rice varieties showed significant effect on $\mathrm{N}$ content in grain but in case of straw it had insignificant effect (Table 1). The highest $\mathrm{N}$ content $(0.727 \%)$ was recorded in BRRI dhan48 and the lowest $N$ content $(0.362 \%)$ was found in NERICA-10. Nitrogen contents in rice grain and straw was significantly influenced by different moisture regimes (Table 1). $\mathrm{N}$ content in grain was increased with the increase of moisture level but the reverse result was observed in case of straw N-content. At $50 \%$ field capacity condition no grain was formed. At flood condition $\mathrm{N}$ content in grain (1.110\%) was recorded highest value and lowest in straw (0.554\%). The highest $\mathrm{N}$ content $(0.576 \%)$ in straw was found at $50 \%$ field capacity condition. The data presented in Table 2 showed significant variations in $\mathrm{N}$ contents of rice grain and straw. The highest $\mathrm{N}$ content $(1.252 \%)$ in grain was found in the treatment combination $\mathrm{As}_{60} \mathrm{~V}_{3} \mathrm{M}_{1}$ which was statistically identical with the treatment combination of $\mathrm{As}_{30} \mathrm{~V}_{1} \mathrm{M}_{2}$ and $\mathrm{As}_{0} \mathrm{~V}_{1} \mathrm{M}_{2}$. No grain was found at $50 \%$ field capacity and $100 \%$ field capacity. Only BRRI dhan48 produced grain at 100\% field capacity condition. Straw N-contents ranged from $0.471 \%$ in the treatment combination $A s_{30} V_{3} M_{2}$ to $0.685 \%$ in the treatment combination $\mathrm{As}_{30} \mathrm{~V}_{3} \mathrm{M}_{0}$.

Phosphorus content: Application of different doses of arsenic to soil resulted significant effect on $P$ content in rice grain and straw (Table 1). Phosphorus content was lowest both in grain $(0.056 \%)$ and straw (0.123\%) when As was applied @ 60 ppm. The highest P content in grain $(0.072 \%)$ was found in the control treatment (no As was applied) which was statistically identical with $30 \mathrm{ppm}$ As treatment. In case of straw the highest P content $(0.136 \%)$ was recorded at $30 \mathrm{ppm}$ As. Phosphorus content of both grain and straw decreased as the dose of As increased. It has been reported that $P$ content in straw decreased with the increase of As doses whereas the reverse result was found for grain-P content (Islam and Jahiruddin, 2010). Phosphorus content in rice grain and straw was significantly affected by different varieties (Table 1$)$. The highest $P$ content both in grain $(0.094 \%)$ and straw $(0.138 \%)$ was recorded in BRRI dhan48 and the lowest $P$ content both in grain $(0.051 \%)$ and straw $(0.125 \%)$ was recorded in NERICA-10. Different moisture regimes had significant effect on $P$ content in rice grain and straw (Table 1). No grain was formed at $50 \%$ field capacity condition. The highest $P$ content in grain $(0.152 \%)$ and in straw $(0.153 \%$ ) were found at flood condition and $50 \%$ field capacity condition, respectively. At $100 \%$ field capacity condition lowest $P$ content $(0.114 \%)$ was found in straw. The interaction effect of different rates of As application, varieties and moisture regime was statistically insignificant on $\mathrm{P}$ contents in rice grain and straw (Table 2). However, numerically highest $P$ content of grain and straw was found in the treatment combination $A s_{30} V_{1} M_{2}$ and $A s_{60} V_{3} M_{0}$, respectively.

Potassium content: Application of different rates of As to soil had significant effect on $\mathrm{K}$ content in rice straw but an insignificant effect was found in case of grain (Table 1). $0.2056 \%, 0.2064 \%$ and $0.1937 \% \mathrm{~K}$ content in grain was recorded at the rate of $0 \mathrm{ppm}, 30 \mathrm{ppm}, 60 \mathrm{ppm}$ As doses, respectively. $\mathrm{K}$ content in straw was highest (1.457\%) with 0 ppm As treatment and the lowest (1.303\%) straw K-content was recorded at $60 \mathrm{ppm}$ As application. Potassium contents in rice grain and straw was influenced by different rice varieties (Table 1 ). BRRI dhan48 resulted the highest $\mathrm{K}$ content both in grain $(0.318 \%)$ and straw $(1.443 \%)$ and the lowest K content in grain $(0.138 \%)$ and straw $(1.293 \%)$ was recorded in NERICA-1. Different moisture condition had significant effect on $\mathrm{K}$ content in rice grain and straw (Table 1). The highest $\mathrm{K}$ content $(0.453 \%)$ in grain and $(1.423 \%)$ in straw were found at flood condition and $50 \%$ field capacity condition, respectively. At $100 \%$ field capacity condition, the lowest $\mathrm{K}$ content (1.317\%) was 
recorded in straw. Potassium content in rice grain showed insignificance to the interaction effect of different dose of As, rice variety and moisture regime whereas this interaction effect was significant on $\mathrm{K}$ content in straw (Table 2). Potassium contents in rice straw ranged from $1.080 \%$ in the treatment combination $\mathrm{As}_{60} \mathrm{~V}_{1} \mathrm{M}_{1}$ to $1.670 \%$ in the treatment combination $\mathrm{As}_{0} \mathrm{~V}_{3} \mathrm{M}_{2}$.

Table 1. Main effects of As, variety and moisture regime on the nutrient contents (\%) of rice

\begin{tabular}{|c|c|c|c|c|c|c|c|c|}
\hline \multirow[t]{2}{*}{ Treatments } & \multicolumn{2}{|c|}{$\mathbf{N}$} & \multicolumn{2}{|c|}{$\mathbf{P}$} & \multicolumn{2}{|c|}{$\mathbf{K}$} & \multicolumn{2}{|l|}{$\mathbf{S}$} \\
\hline & Grain & Straw & Grain & Straw & Grain & Straw & Grain & Straw \\
\hline \multicolumn{9}{|l|}{ Arsenic } \\
\hline $\mathrm{AS}_{0}$ & $0.494 \mathrm{~b}$ & $0.536 \mathrm{c}$ & $0.072 \mathrm{a}$ & $0.134 \mathrm{a}$ & 0.2056 & $1.457 \mathrm{a}$ & $0.047 \mathrm{a}$ & $0.091 \mathrm{a}$ \\
\hline $\mathrm{As}_{30}$ & $0.470 \mathrm{c}$ & $0.563 \mathrm{~b}$ & $0.071 \mathrm{a}$ & $0.136 \mathrm{a}$ & 0.2064 & $1.339 \mathrm{~b}$ & $0.041 \mathrm{~b}$ & $0.087 \mathrm{ab}$ \\
\hline $\mathrm{AS}_{60}$ & $0.515 \mathrm{a}$ & $0.592 \mathrm{a}$ & $0.056 \mathrm{~b}$ & $0.123 \mathrm{~b}$ & 0.1937 & $1.303 \mathrm{c}$ & $0.040 \mathrm{~b}$ & $0.084 \mathrm{~b}$ \\
\hline Level of significance & $\star \star$ & $* *$ & $\star *$ & ** & NS & ** & ** & $* *$ \\
\hline CV (\%) & 7.05 & 4.74 & 14.93 & 8.84 & 13.22 & 4.09 & 8.02 & 6.15 \\
\hline \multicolumn{9}{|l|}{ Variety } \\
\hline NERICA-1 $\left(V_{1}\right)$ & $0.390 \mathrm{~b}$ & 0.564 & $0.054 \mathrm{~b}$ & $0.131 \mathrm{~b}$ & $0.138 \mathrm{~b}$ & $1.293 \mathrm{C}$ & $0.030 \mathrm{~b}$ & $0.084 \mathrm{~b}$ \\
\hline NERICA-10 $\left(\mathrm{V}_{2}\right)$ & $0.362 \mathrm{c}$ & 0.556 & $0.051 \mathrm{~b}$ & $0.125 \mathrm{~b}$ & $0.149 \mathrm{~b}$ & $1.363 \mathrm{~b}$ & $0.031 \mathrm{~b}$ & $0.088 \mathrm{ab}$ \\
\hline BRRIdhan48 $\left(\mathrm{V}_{3}\right)$ & $0.727 \mathrm{a}$ & 0.570 & $0.094 \mathrm{a}$ & $0.138 \mathrm{a}$ & $0.318 \mathrm{a}$ & $1.443 \mathrm{a}$ & $0.065 \mathrm{a}$ & $0.091 \mathrm{a}$ \\
\hline Level of significance & $\star \star *$ & NS & ** & ** & ** & ** & ** & $\star \star *$ \\
\hline CV (\%) & 7.05 & 4.74 & 14.93 & 8.84 & 13.22 & 4.09 & 8.02 & 6.15 \\
\hline \multicolumn{9}{|l|}{ Moisture regime } \\
\hline $50 \% \mathrm{FC}\left(\mathrm{M}_{0}\right)$ & - & $0.576 \mathrm{a}$ & - & $0.153 \mathrm{a}$ & - & $1.423 \mathrm{a}$ & - & $0.085 \mathrm{~b}$ \\
\hline $100 \% \mathrm{FC}\left(\mathrm{M}_{1}\right)$ & $0.369 \mathrm{~b}$ & $0.559 \mathrm{ab}$ & $0.047 \mathrm{~b}$ & $0.114 \mathrm{c}$ & $0.153 \mathrm{~b}$ & $1.317 \mathrm{c}$ & $0.034 \mathrm{~b}$ & $0.093 \mathrm{a}$ \\
\hline Flood $\left(\mathrm{M}_{2}\right)$ & $1.110 \mathrm{a}$ & $0.554 \mathrm{~b}$ & $0.152 \mathrm{a}$ & $0.126 \mathrm{~b}$ & $0.453 \mathrm{a}$ & $1.360 \mathrm{~b}$ & $0.092 \mathrm{a}$ & $0.083 \mathrm{~b}$ \\
\hline Level of significance & $\star \star$ & $\star \star$ & $\star \star *$ & $\star \star *$ & $\star \star$ & ** & ** & $\star \star *$ \\
\hline CV (\%) & 7.05 & 4.74 & 14.93 & 8.84 & 13.22 & 4.09 & 8.02 & 6.15 \\
\hline
\end{tabular}

In a column, figures with same letter(s) or without letter do not differ significantly whereas figures with different letter(s) differ significantly at $1 \%$ level of significance

Table 2. Interaction effects of As, variety and moisture regime on the nutrient contents (\%) of rice

\begin{tabular}{|c|c|c|c|c|c|c|c|c|}
\hline \multirow{2}{*}{$\begin{array}{l}\text { Treatments } \\
\text { (Arsenic x Variety } \\
\text { x Moisture) }\end{array}$} & \multicolumn{2}{|c|}{$\mathbf{N}$} & \multicolumn{2}{|c|}{$\mathbf{P}$} & \multicolumn{2}{|c|}{$\mathrm{K}$} & \multicolumn{2}{|c|}{ S } \\
\hline & Grain & Straw & Grain & Straw & Grain & Straw & Grain & Straw \\
\hline \multirow{3}{*}{$\begin{array}{l}A s_{0} V_{1} M_{0} \\
A s_{0} V_{1} M_{1} \\
A s_{0} V_{1} M_{2}\end{array}$} & - & $0.532 \mathrm{~h}-\mathrm{I}$ & - & 0.124 & - & $1.237 \mathrm{gh}$ & - & 0.087 \\
\hline & - & $0.564 \mathrm{e}-\mathrm{j}$ & - & 0.126 & - & $1.180 \mathrm{~h}$ & - & 0.090 \\
\hline & $1.217 \mathrm{ab}$ & $0.609 b-f$ & 0.171 & 0.142 & 0.412 & $1.237 \mathrm{gh}$ & $0.101 \mathrm{abc}$ & 0.092 \\
\hline $\mathrm{As}_{0} \mathrm{~V}_{2} \mathrm{M}_{0}$ & - & $0.475 \mathrm{Im}$ & - & 0.144 & - & $1.193 \mathrm{~h}$ & - & 0.085 \\
\hline $\mathrm{As}_{0} \mathrm{~V}_{2} \mathrm{M}_{1}$ & - & $0.509 \mathrm{i}-\mathrm{m}$ & - & 0.113 & - & $1.330 \mathrm{fg}$ & - & 0.104 \\
\hline $\mathrm{As}_{0} \mathrm{~V}_{2} \mathrm{M}_{2}$ & $1.010 \mathrm{f}$ & 0.504 j-m & 0.178 & 0.123 & 0.473 & $1.430 \mathrm{de}$ & $0.097 \mathrm{abc}$ & 0.088 \\
\hline $\mathrm{As}_{0} \mathrm{~V}_{3} \mathrm{M}_{0}$ & - & $0.523 \mathrm{~h}-\mathrm{m}$ & - & 0.166 & - & $1.223 \mathrm{~h}$ & - & 0.089 \\
\hline $\mathrm{As}_{0} \mathrm{~V}_{3} \mathrm{M}_{1}$ & $1.108 \mathrm{de}$ & $0.554 \mathrm{f}-\mathrm{j}$ & 0.150 & 0.125 & 0.465 & $1.400 \mathrm{~d}-\mathrm{f}$ & $0.096 \mathrm{abc}$ & 0.098 \\
\hline $\mathrm{As}_{0} \mathrm{~V}_{3} \mathrm{M}_{2}$ & 1.114 cde & $0.557 \mathrm{e}-\mathrm{j}$ & 0.151 & 0.145 & 0.499 & $1.670 \mathrm{a}$ & $0.107 \mathrm{a}$ & 0.087 \\
\hline $\mathrm{As}_{30} \mathrm{~V}_{1} \mathrm{M}_{0}$ & - & $0.539 \mathrm{~h}-\mathrm{k}$ & - & 0.143 & - & 1.477 b-e & - & 0.087 \\
\hline $\mathrm{As}_{30} \mathrm{~V}_{1} \mathrm{M}_{1}$ & - & $0.567 \mathrm{~d}-\mathrm{i}$ & - & 0.110 & - & $1.490 \mathrm{a}-\mathrm{d}$ & - & 0.088 \\
\hline $\mathrm{As}_{30} \mathrm{~V}_{1} \mathrm{M}_{2}$ & $1.232 \mathrm{a}$ & 0.616 b-e & 0.185 & 0.154 & 0.413 & $1.240 \mathrm{gh}$ & $0.094 \mathrm{abc}$ & 0.086 \\
\hline $\mathrm{AS}_{30} \mathrm{~V}_{2} \mathrm{M}_{0}$ & - & $0.602 \mathrm{~b}-\mathrm{g}$ & - & 0.146 & - & $1.55 \mathrm{a}-\mathrm{c}$ & - & 0.075 \\
\hline $\mathrm{As}_{30} \mathrm{~V}_{2} \mathrm{M}_{1}$ & - & $0.561 \mathrm{e}-\mathrm{j}$ & - & 0.115 & - & $1.440 \mathrm{de}$ & - & 0.098 \\
\hline $\mathrm{As}_{30} \mathrm{~V}_{2} \mathrm{M}_{2}$ & $1.092 \mathrm{e}$ & $0.546 \mathrm{~g}-\mathrm{j}$ & 0.152 & 0.127 & 0.458 & $1.377 \mathrm{ef}$ & $0.085 \mathrm{bc}$ & 0.077 \\
\hline $\mathrm{As}_{30} \mathrm{~V}_{3} \mathrm{M}_{0}$ & - & $0.685 \mathrm{a}$ & - & 0.170 & - & $1.580 \mathrm{ab}$ & - & 0.098 \\
\hline $\mathrm{As}_{30} \mathrm{~V}_{3} \mathrm{M}_{1}$ & $0.963 \mathrm{fg}$ & $0.481 \mathrm{k}-\mathrm{m}$ & 0.143 & 0.125 & 0.494 & $1.483 \mathrm{~b}-\mathrm{d}$ & $0.102 \mathrm{ab}$ & 0.093 \\
\hline $\mathrm{As}_{30} \mathrm{~V}_{3} \mathrm{M}_{2}$ & $0.942 \mathrm{~g}$ & $0.471 \mathrm{~m}$ & 0.161 & 0.134 & 0.492 & $1.477 \mathrm{c}-\mathrm{e}$ & 0.088 bc & 0.080 \\
\hline $\mathrm{AS}_{60} \mathrm{~V}_{1} \mathrm{M}_{0}$ & - & $0.579 \mathrm{c}-\mathrm{h}$ & - & 0.154 & - & $1.450 \mathrm{c}-\mathrm{e}$ & - & 0.074 \\
\hline $\mathrm{As}_{60} \mathrm{~V}_{1} \mathrm{M}_{1}$ & - & $0.542 \mathrm{~g}-\mathrm{j}$ & - & 0.107 & - & $1.080 \mathrm{i}$ & - & 0.080 \\
\hline $\mathrm{AS}_{60} \mathrm{~V}_{1} \mathrm{M}_{2}$ & $1.064 \mathrm{e}$ & 0.528 h-m & 0.135 & 0.113 & 0.416 & $1.250 \mathrm{gh}$ & $0.082 \mathrm{c}$ & 0.071 \\
\hline $\mathrm{As}_{60} \mathrm{~V}_{2} \mathrm{M}_{0}$ & - & $0.602 \mathrm{~b}-\mathrm{g}$ & - & 0.151 & - & $1.503 \mathrm{a}-\mathrm{d}$ & - & 0.082 \\
\hline $\mathrm{As}_{60} \mathrm{~V}_{2} \mathrm{M}_{1}$ & - & $0.635 \mathrm{a}-\mathrm{c}$ & - & 0.105 & - & $1.190 \mathrm{~h}$ & - & 0.102 \\
\hline $\mathrm{As}_{60} \mathrm{~V}_{2} \mathrm{M}_{2}$ & $1.156 \mathrm{~cd}$ & $0.578 \mathrm{c}-\mathrm{h}$ & 0.126 & 0.105 & 0.416 & $1.253 \mathrm{gh}$ & $0.091 \mathrm{abc}$ & 0.083 \\
\hline $\mathrm{As}_{60} \mathrm{~V}_{3} \mathrm{M}_{0}$ & - & $0.655 a b$ & 0.000 & 0.178 & - & $1.590 \mathrm{a}$ & - & 0.093 \\
\hline $\mathrm{As}_{60} \mathrm{~V}_{3} \mathrm{M}_{1}$ & $1.252 \mathrm{a}$ & $0.626 \mathrm{~b}-\mathrm{d}$ & 0.126 & 0.105 & 0.418 & $1.257 \mathrm{gh}$ & $0.099 \mathrm{abc}$ & 0.090 \\
\hline $\mathrm{As}_{60} \mathrm{~V}_{3} \mathrm{M}_{2}$ & $1.167 \mathrm{bc}$ & $0.583 \mathrm{c}-\mathrm{h}$ & 0.114 & 0.094 & 0.491 & $1.477 \mathrm{~b}-\mathrm{e}$ & $0.094 \mathrm{abc}$ & 0.085 \\
\hline Level of significance & ** & ** & NS & NS & NS & $\star \star$ & $\star \star$ & NS \\
\hline CV (\%) & 7.05 & 4.74 & 14.93 & 8.84 & 13.22 & 4.09 & 8.02 & 6.15 \\
\hline
\end{tabular}

In a column, figures with same letter(s) or without letter do not differ significantly whereas figures with different letter(s) differ significantly at $1 \%$ level of significance. $V_{1}=$ NERICA-1, $V_{2}=$ NERICA-10, $V_{3}=B R R I$ dhan $48, M_{0}=50 \%$ field capacity, $M_{1}=100 \%$ field capacity, $M_{2}=$ Flood condition 
Sulphur content: Sulphur concentrations of both grain and straw decreased with increasing concentration of arsenic, the range being $0.040-0.047 \% \mathrm{~S}$ for grain and $0.084-0.091 \% \mathrm{~S}$ for straw (Table 1). Variety had significant effects on $S$ contents in rice (Table 1). The highest $S$ content was found both in grain (0.065\%) and straw (0.091\%) of BRRI dhan48 and NERICA-1 recorded lowest $S$ content in grain $(0.030 \%)$ and straw $(0.084 \%)$. Moisture regime considerably affected $\mathrm{S}$ contents in rice (Table 1$)$. The highest $S$ content $0.092 \%$ in grain and $0.093 \%$ in straw was found at flood condition and $100 \%$ field capacity, respectively. At flood condition the lowest S content $(0.083 \%)$ was recorded in straw. Table 2 illustrates that the combined interaction effect of different doses of As, rice varieties and moisture regime on $\mathrm{S}$ content in rice grain and it was found significant but straw S-content was not influenced by this effect. The highest $S$ content in grain (0.107\%) was found in the treatment combination $\mathrm{As}_{0} \mathrm{~V}_{3} \mathrm{M}_{2}$.

Nitrogen uptake: Increasing doses of As significantly decreased $\mathrm{N}$ uptake by grain and straw (Table 3). The maximum $\mathrm{N}$ uptake $\left(22.14 \mathrm{mg} \mathrm{pot}^{-1}\right)$ by grain and $\left(45.41 \mathrm{mg} \mathrm{pot}^{-1}\right)$ by straw was recorded at $0 \mathrm{ppm}$ As treatment whereas minimum $\mathrm{N}$ uptake $\left(11.26 \mathrm{mg} \mathrm{pot}^{-1}\right.$ by grain and $31.86 \mathrm{mg} \mathrm{pot}^{-1}$ by straw) was found at $60 \mathrm{ppm}$ As treatment. The highest $\mathrm{N}$ uptake by grain $\left(27.28 \mathrm{mg} \mathrm{pot}^{-1}\right)$ and straw $\left(38.96 \mathrm{mg} \mathrm{pot}^{-1}\right)$ was found in BRRI dhan48 (Table 3). The lowest $\mathrm{N}$ uptake by grain (12.59 $\left.\mathrm{mg} \mathrm{pot}^{-1}\right)$ and straw (27.44 mg pot $^{-1}$ ) was found in NERICA-1. Nitrogen uptake by grain and straw was significantly increased with the increase of moisture regime (Table 3). Maximum $N$ uptake $\left(50.49 \mathrm{mg} \mathrm{pot}^{-1}\right)$ and $\left(57.86 \mathrm{mg} \mathrm{pot}^{-1}\right)$ was observed at flood condition in grain and straw, respectively. The lowest $N$ uptake $\left(31.14 \mathrm{mg} \mathrm{pot}^{-1}\right)$ in straw was recorded at $50 \%$ field capacity condition, where no grain was found at this moisture condition. Nitrogen uptake in grain and straw was significantly influenced by the interaction of As, varieties and different moisture regime (Table 4). Maximum $N$ uptake (161.61 $\left.\mathrm{mg} \mathrm{pot}^{-1}\right)$ in grain and (112.92 mg pot ${ }^{-1}$ ) straw was observed in the treatment combination $\mathrm{As}_{0} \mathrm{~V}_{3} \mathrm{M}_{2}$. The lowest $\mathrm{N}$ uptake in straw (11.22 $\left.\mathrm{mg} \mathrm{pot}^{-1}\right)$ was recorded in the treatment combination $\mathrm{As}_{60} \mathrm{~V}_{1} \mathrm{M}_{0}$.

Phosphorus uptake: Phosphorus uptake was significantly decreased with the increase of added As doses (Table 3). The highest $P$ uptake by both grain $\left(3.95 \mathrm{mg} \mathrm{pot}^{-1}\right)$ and straw $\left(10.86 \mathrm{mg} \mathrm{pot}^{-1}\right)$ was found when no As was added. The lowest $P$ uptake by grain (1.69 mg pot $\left.{ }^{-1}\right)$ and straw (6.69 $\left.\mathrm{mg} \mathrm{pot}^{-1}\right)$ was observed in $60 \mathrm{ppm}$ As. The highest $P$ uptake by grain $\left(3.83 \mathrm{mg} \mathrm{pot}^{-1}\right)$ and straw $\left(10.68 \mathrm{mg} \mathrm{pot}^{-1}\right)$ was found in BRRI dhan48 (Table 3). Again, the lowest $P$ uptake by grain $\left(2.20 \mathrm{mg} \mathrm{pot}^{-1}\right)$ and straw (6.59 mg pot $^{-1}$ ) was found in NERICA-10. Phosphorus uptake by grain and straw was significantly increased with the increase of moisture regime (Table 4). Maximum $P$ uptake $\left(6.59 \mathrm{mg} \mathrm{pot}^{-1}\right.$ and $13.46 \mathrm{mg} \mathrm{pot}^{-1}$ ) was observed in the flood condition in grain and straw, respectively. Minimum $\mathrm{P}$ uptake $\left(4.85 \mathrm{mg} \mathrm{pot}^{-1}\right)$ in straw was recorded at $50 \%$ field capacity condition when no grain was found at this moisture condition. Phosphorus uptake by rice was significantly influenced by the interaction effects of added As, variety and moisture regime (Table 4). Maximum $P$ uptake $\left(30.57 \mathrm{mg} \mathrm{pot}^{1}\right)$ by grain and $\left(30.63 \mathrm{mg} \mathrm{pot}^{-1}\right)$ straw was observed in the treatment combination $\mathrm{As}_{0} \mathrm{~V}_{3} \mathrm{M}_{2}$. Minimum $\mathrm{P}$ uptake in straw (2.64 mg pot ${ }^{1}$ ) was recorded in the treatment combination $\mathrm{As}_{60} \mathrm{~V}_{2} \mathrm{M}_{0}$.

Potassium uptake: Increased doses of As remarkably decreased $\mathrm{K}$ uptake by rice (Table 3). Maximum $\mathrm{K}$ uptake $\left(17.76 \mathrm{mg} \mathrm{pot}^{-1}\right)$ by grain and $\left(104.44 \mathrm{mg} \mathrm{pot}^{-1}\right)$ by straw was recorded at $0 \mathrm{ppm}$ As treatment. The minimum K uptake in grain (6.64 $\mathrm{mg} \mathrm{pot}^{-1}$ ) and straw $\left(50.50 \mathrm{mg} \mathrm{pot}^{-1}\right.$ ) were observed in $60 \mathrm{ppm} \mathrm{As}$ treatment. The highest $\mathrm{K}$ uptake by grain $\left(15.73 \mathrm{mg} \mathrm{pot}^{-1}\right)$ and straw (114.31 $\mathrm{mg} \mathrm{pot}^{-1}$ ) was found in BRRI dhan48 (Table 3). The Lowest $\mathrm{K}$ uptake by grain (11.39 $\mathrm{mg} \mathrm{pot}^{-1}$ ) and straw (70.88 $\mathrm{mg} \mathrm{pot}^{-1}$ ) was observed in NERICA-10. Potassium uptake by grain and straw was significantly increased with the increase of moisture level (Table 3). Maximum K uptake by grain (30.14 $\mathrm{mg} \mathrm{pot}^{-1}$ ) and straw (121.82 mg pot $^{-1}$ ) was observed in the flood condition. The lowest K uptake (45.69 $\left.\mathrm{mg} \mathrm{pot}^{-1}\right)$ in straw was recorded at $50 \%$ field capacity condition when no grain was found at this moisture condition. Grain yield was not found at $50 \%$ and $100 \%$ field capacity condition except BRRI dhan48 which produced grain at $100 \%$ field capacity condition and a significant interaction effect was observed on $\mathrm{K}$ uptake by rice (Table 4). Maximum K uptake $\left(74.04 \mathrm{mg} \mathrm{pot}^{-1}\right)$ by grain and $\left(227.31 \mathrm{mg} \mathrm{pot}^{-1}\right)$ by straw was observed in the treatment combination $\mathrm{As}_{0} \mathrm{~V}_{3} \mathrm{M}_{2}$. Minimum $\mathrm{K}$ uptake $\left(29.70 \mathrm{mg} \mathrm{pot}^{-1}\right.$ ) by straw was recorded in the treatment combination $\mathrm{As}_{60} \mathrm{~V}_{2} \mathrm{M}_{0}$. 
Table 3. Main effects of As, variety and moisture regime on the nutrient uptake $\left(\mathrm{mg} \mathrm{pot}^{-1}\right)$ by rice

\begin{tabular}{|c|c|c|c|c|c|c|c|c|}
\hline \multirow[t]{2}{*}{ Treatments } & \multicolumn{2}{|c|}{$\mathbf{N}$} & \multicolumn{2}{|c|}{$\mathbf{P}$} & \multicolumn{2}{|c|}{$\mathrm{K}$} & \multicolumn{2}{|c|}{$\mathbf{S}$} \\
\hline & Grain & Straw & Grain & Straw & Grain & Straw & Grain & Straw \\
\hline \multicolumn{9}{|l|}{ Arsenic } \\
\hline $\mathrm{AS}_{0}$ & $22.14 a$ & $45.41 a$ & $3.95 a$ & $10.86 a$ & $17.76 \mathrm{a}$ & $104.44 a$ & $4.98 \mathrm{a}$ & $7.23 a$ \\
\hline $\mathrm{AS}_{30}$ & $16.44 b$ & $41.30 \mathrm{~b}$ & $2.85 b$ & $8.21 \mathrm{~b}$ & $10.56 \mathrm{~b}$ & $62.92 \mathrm{~b}$ & $2.66 \mathrm{~b}$ & $5.26 \mathrm{~b}$ \\
\hline $\mathrm{As}_{60}$ & $11.26 \mathrm{c}$ & $31.86 \mathrm{c}$ & $1.69 \mathrm{c}$ & $6.69 \mathrm{c}$ & $6.64 c$ & $50.50 \mathrm{c}$ & $1.68 \mathrm{c}$ & $3.66 \mathrm{c}$ \\
\hline CV(\%) & 9.334 & 6.668 & 9.934 & 7.209 & 10.420 & 7.454 & 9.577 & 6.726 \\
\hline Level of sig. & $\star \star$ & $\star \star$ & $\star \star$ & $\star \star$ & $\star \star$ & $\star \star$ & $\star \star$ & $\star \star$ \\
\hline \multicolumn{9}{|l|}{ Variety } \\
\hline NERICA-1 $\left(V_{1}\right)$ & $12.59 \mathrm{~b}$ & $27.44 \mathrm{~b}$ & $2.23 b$ & $6.65 b$ & $12.40 \mathrm{~b}$ & $72.36 \mathrm{~b}$ & $1.73 \mathrm{~b}$ & $4.41 b$ \\
\hline NERICA-10 $\left(V_{2}\right)$ & $13.96 \mathrm{~b}$ & $30.36 \mathrm{~b}$ & $2.20 \mathrm{~b}$ & $6.59 \mathrm{~b}$ & $11.39 \mathrm{~b}$ & $70.88 \mathrm{~b}$ & $1.77 \mathrm{~b}$ & $4.70 \mathrm{~b}$ \\
\hline BRRIdhan48 $\left(\mathrm{V}_{3}\right)$ & $27.28 \mathrm{a}$ & $38.96 a$ & $3.83 a$ & $10.68 \mathrm{a}$ & $15.73 a$ & $114.31 \mathrm{a}$ & $2.98 \mathrm{a}$ & $7.11 \mathrm{a}$ \\
\hline $\mathrm{CV}(\%)$ & 9.334 & 6.668 & 9.934 & 7.209 & 10.420 & 7.454 & 9.577 & 6.726 \\
\hline Level of sig. & $\star *$ & * & $\star *$ & $\star *$ & $\star *$ & $\star *$ & $\star *$ & $\star *$ \\
\hline \multicolumn{9}{|l|}{ Moisture regime } \\
\hline $50 \% \mathrm{FC}\left(\mathrm{M}_{0}\right)$ & - & $31.14 \mathrm{~b}$ & - & $4.85 b$ & - & $45.69 \mathrm{c}$ & - & $3.48 \mathrm{c}$ \\
\hline $100 \% \mathrm{FC}\left(\mathrm{M}_{1}\right)$ & $7.60 \mathrm{~b}$ & $34.68 \mathrm{~b}$ & $1.36 \mathrm{~b}$ & $7.08 \mathrm{~b}$ & $5.19 b$ & $63.36 \mathrm{~b}$ & $1.79 \mathrm{~b}$ & $4.65 b$ \\
\hline Flood $\left(\mathrm{M}_{2}\right)$ & $50.49 a$ & $57.86 a$ & $5.76 a$ & $13.46 a$ & $30.14 a$ & $121.82 \mathrm{a}$ & $6.90 \mathrm{a}$ & $9.32 \mathrm{a}$ \\
\hline $\mathrm{CV}(\%)$ & 9.334 & 6.668 & 9.934 & 7.209 & 10.420 & 7.454 & 9.577 & 6.726 \\
\hline Level of sig. & ** & $\star \star$ & $\star \star$ & $\star \star$ & $\star *$ & $\star \star$ & ** & $\star *$ \\
\hline
\end{tabular}

In a column, figures with same letter(s) or without letter do not differ significantly whereas figures with different letter(s) differ significantly at $1 \%$ level of significance

Table 4. Interaction effects of As, variety and moisture regime on the nutrient uptake $\left(\mathrm{mg} \mathrm{pot}^{-1}\right)$ by rice

\begin{tabular}{|c|c|c|c|c|c|c|c|c|}
\hline \multirow{2}{*}{$\begin{array}{l}\text { Treatments } \\
\text { (Arsenic x Variety x } \\
\text { Moisture) }\end{array}$} & \multicolumn{2}{|c|}{$\mathbf{N}$} & \multicolumn{2}{|c|}{$\mathbf{P}$} & \multicolumn{2}{|c|}{$\mathrm{K}$} & \multicolumn{2}{|c|}{$\mathbf{S}$} \\
\hline & Grain & Straw & Grain & Straw & Grain & Straw & Grain & Straw \\
\hline $\mathrm{As}_{0} \mathrm{~V}_{1} \mathrm{M}_{0}$ & - & $20.28 \mathrm{~g}$ & - & $5.28 \mathrm{fg}$ & - & $42.7 \mathrm{~h}$ & - & $3.38 f$ \\
\hline $\mathrm{As}_{0} \mathrm{~V}_{1} \mathrm{M}_{1}$ & - & $27.56 f$ & - & $7.80 \mathrm{f}$ & - & $57.21 \mathrm{f}$ & - & 4.94de \\
\hline $\mathrm{As}_{0} \mathrm{~V}_{1} \mathrm{M}_{2}$ & $63.09 \mathrm{c}$ & $71.92 \mathrm{c}$ & $13.33 \mathrm{c}$ & $17.55 \mathrm{c}$ & $22.17 d$ & $161.80 \mathrm{bc}$ & $8.84 \mathrm{~cd}$ & $11.13 b$ \\
\hline $\mathrm{As}_{0} \mathrm{~V}_{2} \mathrm{M}_{0}$ & - & 19.72gh & - & $5.52 f$ & - & $41.44 \mathrm{~h}$ & - & $3.29 f$ \\
\hline \multirow{3}{*}{$\begin{array}{l}A s_{0} V_{2} M_{1} \\
A s_{0} V_{2} M_{2} \\
A s_{0} V_{3} M_{0}\end{array}$} & - & $26.68 f$ & - & $7.64 f$ & - & $58.10 \mathrm{f}$ & - & $4.49 \mathrm{e}$ \\
\hline & $57.12 \mathrm{c}$ & $61.20 c$ & $13.43 \mathrm{c}$ & $15.23 d$ & $25.29 d$ & $141.40 \mathrm{c}$ & $8.83 \mathrm{~cd}$ & $9.38 c$ \\
\hline & - & $20.80 \mathrm{~g}$ & - & $6.36 f$ & - & 43.56gh & - & $3.47 f$ \\
\hline $\mathrm{As}_{0} \mathrm{~V}_{3} \mathrm{M}_{1}$ & $45.01 d$ & $34.45 \mathrm{e}$ & $6.11 d$ & $9.51 \mathrm{e}$ & $10.65 \mathrm{fg}$ & $73.16 \mathrm{e}$ & $3.67 d$ & $6.27 \mathrm{~d}$ \\
\hline $\mathrm{As}_{0} \mathrm{~V}_{3} \mathrm{M}_{2}$ & $161.61 \mathrm{a}$ & $112.92 \mathrm{a}$ & $30.57 a$ & $30.63 a$ & $74.04 a$ & $227.31 \mathrm{a}$ & $17.61 \mathrm{a}$ & $17.29 \mathrm{a}$ \\
\hline \multirow{3}{*}{$\begin{array}{l}\mathrm{As}_{30} \mathrm{~V}_{1} \mathrm{M}_{0} \\
\mathrm{As}_{30} \mathrm{~V}_{1} \mathrm{M}_{1} \\
\mathrm{As}_{30} \mathrm{~V}_{1} \mathrm{M}_{2}\end{array}$} & - & $16.20 \mathrm{~h}$ & - & $3.96 \mathrm{~h}$ & - & $34.72 \mathrm{i}$ & - & $2.81 \mathrm{fg}$ \\
\hline & - & $26.18 f$ & - & $5.67 f$ & - & $52.65 \mathrm{fg}$ & - & $4.08 \mathrm{e}$ \\
\hline & $43.13 d$ & $51.17 d$ & $6.09 d$ & $11.08 \mathrm{e}$ & $16.16 \mathrm{e}$ & $93.68 \mathrm{~d}$ & 3.06de & $7.48 \mathrm{c}$ \\
\hline \multirow{2}{*}{$\begin{array}{l}\mathrm{As}_{30} \mathrm{~V}_{2} \mathrm{M}_{0} \\
\mathrm{As}_{30} \mathrm{~V}_{2} \mathrm{M}_{1}\end{array}$} & - & $16.80 \mathrm{~h}$ & - & $4.18 \mathrm{~g}$ & - & $35.84 i$ & - & $2.63 f g$ \\
\hline & - & $24.06 \mathrm{fg}$ & - & $5.52 f$ & - & $52.64 \mathrm{fg}$ & - & $4.03 \mathrm{e}$ \\
\hline $\mathrm{As}_{30} \mathrm{~V}_{2} \mathrm{M}_{2}$ & $40.16 d$ & $50.84 d$ & 5.61de & $9.83 \mathrm{e}$ & $16.20 \mathrm{e}$ & $91.23 d$ & $2.96 \mathrm{e}$ & $7.30 \mathrm{c}$ \\
\hline \multirow{2}{*}{$\begin{array}{l}\mathrm{As}_{30} \mathrm{~V}_{3} \mathrm{M}_{0} \\
\mathrm{As}_{30} \mathrm{~V}_{3} \mathrm{M}_{1}\end{array}$} & - & $15.83 \mathrm{~h}$ & - & $4.43 \mathrm{~g}$ & - & $36.64 i$ & - & $2.72 \mathrm{fg}$ \\
\hline & $25.66 f$ & $30.25 \mathrm{e}$ & $4.04 \mathrm{e}$ & $7.36 f$ & $7.86 \mathrm{~g}$ & 64.69ef & $2.50 \mathrm{e}$ & $5.45 d$ \\
\hline $\mathrm{As}_{30} \mathrm{~V}_{3} \mathrm{M}_{2}$ & $130.66 b$ & $93.35 b$ & $27.88 \mathrm{~b}$ & $21.88 \mathrm{~b}$ & $52.02 \mathrm{~b}$ & $186.74 b$ & $10.18 \mathrm{~b}$ & $13.68 \mathrm{~b}$ \\
\hline $\mathrm{As}_{60} \mathrm{~V}_{1} \mathrm{M}_{0}$ & - & $11.22 \mathrm{i}$ & - & $3.05 \mathrm{~h}$ & - & $30.80 \mathrm{ij}$ & - & $2.06 \mathrm{~h}$ \\
\hline $\mathrm{As}_{60} \mathrm{~V}_{1} \mathrm{M}_{1}$ & - & $18.80 \mathrm{~g}$ & - & $3.96 \mathrm{~h}$ & - & $48.16 \mathrm{~g}$ & - & $3.44 f$ \\
\hline $\mathrm{AS}_{60} \mathrm{~V}_{1} \mathrm{M}_{2}$ & $35.10 \mathrm{e}$ & $50.09 \mathrm{~d}$ & $6.14 d$ & $7.04 \mathrm{f}$ & $12.60 \mathrm{f}$ & $71.50 \mathrm{e}$ & $2.29 \mathrm{e}$ & $5.53 d$ \\
\hline $\mathrm{As}_{60} \mathrm{~V}_{2} \mathrm{M}_{0}$ & - & 14.12hi & - & $2.64 \mathrm{i}$ & - & $29.70 j$ & - & $2.02 \mathrm{~h}$ \\
\hline $\mathrm{AS}_{60} \mathrm{~V}_{2} \mathrm{M}_{1}$ & - & $21.16 \mathrm{~g}$ & - & $3.61 \mathrm{~h}$ & - & $47.74 \mathrm{~g}$ & - & $3.53 f$ \\
\hline \multirow{2}{*}{$\begin{array}{l}A s_{60} V_{2} M_{2} \\
A s_{60} V_{3} M_{0}\end{array}$} & $30.30 \mathrm{e}$ & 48.96de & $6.07 d$ & $6.83 f$ & $12.15 f$ & $68.20 \mathrm{e}$ & $2.07 \mathrm{ef}$ & $5.35 d$ \\
\hline & - & 15.43h & - & $3.57 \mathrm{~h}$ & - & $31.90 \mathrm{i}$ & - & $2.63 \mathrm{~g}$ \\
\hline $\mathrm{As}_{60} \mathrm{~V}_{3} \mathrm{M}_{1}$ & $22.16 f$ & $28.02 f$ & $3.05 f$ & $5.94 f$ & $7.09 \mathrm{~g}$ & $61.60 f$ & $1.67 f$ & $5.19 \mathrm{~d}$ \\
\hline $\mathrm{As}_{60} \mathrm{~V}_{3} \mathrm{M}_{2}$ & $109.08 b$ & $86.95 b$ & $12.55 \mathrm{c}$ & $13.97 d$ & $40.18 \mathrm{c}$ & $156.73 c$ & $9.12 \mathrm{c}$ & $8.33 c$ \\
\hline CV(\%) & 9.34 & 6.668 & 9.934 & 7.209 & 10.420 & 7.454 & 9.577 & 6.726 \\
\hline Level of sig. & $\star *$ & $\star *$ & $\star *$ & $\star *$ & $\star *$ & $\star \star$ & $\star \star$ & $\star \star$ \\
\hline
\end{tabular}

In a column, figures with same letter(s) or without letter do not differ significantly whereas figures with different letter(s) differ significantly at $1 \%$ level of significance. $\mathrm{V}_{1}=$ NERICA-1, $\mathrm{V}_{2}=$ NERICA-10, $\mathrm{V}_{3}=$ BRRI dhan $48, \mathrm{M}_{0}=50 \%$ field capacity, $\mathrm{M}_{1}=100 \%$ field capacity, $\mathrm{M}_{2}=$ Flood condition 
Sulphur uptake: Sulphur uptake by grain and straw decreased with the increase dose of arsenic, the range being (1.68-4.98 $\mathrm{mg} \mathrm{pot}^{-1}$ ) by grain and (3.66-7.23 mg pot ${ }^{-1}$ ) by straw (Table 3). Variety had significant effects on $\mathrm{S}$ uptake by rice (Table 3). Maximum $\mathrm{S}$ uptake by grain $\left(2.98 \mathrm{mg} \mathrm{pot}^{-1}\right)$ and straw $\left(7.11 \mathrm{mg} \mathrm{pot}^{-1}\right)$ was found in BRRI dhan48 and NERICA-1 recorded minimum $\mathrm{S}$ uptake by grain $(1.73 \mathrm{mg}$ pot $^{-1}$ ) and straw $\left(4.41 \mathrm{mg} \mathrm{pot}^{-1}\right)$. Moisture regime considerably affected $\mathrm{S}$ uptake by rice (Table 3$)$. There was no grain formation at $50 \%$ field capacity. The maximum S uptake $\left(6.90 \mathrm{mg} \mathrm{pot}^{-1}\right)$ by grain and $(9.32$ $\mathrm{mg} \mathrm{pot}^{-1}$ ) by straw was found at flood condition. At $50 \%$ field capacity, minimum S uptake (3.48 $\left.\mathrm{mg} \mathrm{pot}^{-1}\right)$ was recorded in straw. Table 2 illustrates that the combined interaction effect of different doses of As, rice varieties and moisture regime on $\mathrm{S}$ uptake by rice grain and was found significant. The maximum $\mathrm{S}$ uptake by grain $\left(17.21 \mathrm{mg} \mathrm{pot}^{-1}\right)$ and straw $\left(17.29 \mathrm{mg} \mathrm{pot}^{-1}\right)$ were found in the treatment combination $\mathrm{As}_{0} \mathrm{~V}_{3} \mathrm{M}_{2}$.

\section{Conclusion}

It appears that higher level of As in soil adversely affected the nutrient content as well as uptake by rice. With the increase of moisture regime nutrient uptake was increased. At flood condition all the rice varieties recorded higher nutrient content and uptake by both grain and straw compared to other two level of moisture irrespective of As dose. Although, P, K, S contents both in grain and straw decreased with the increasing As level but $\mathrm{N}$ content in rice showed opposite trend. Grain was not obtained from plants grown under $50 \%$ and $100 \%$ field capacity condition apart from variety BRRI dhan48 which produced grain at $100 \%$ field capacity condition. The treatment combination $\mathrm{As}_{0} \mathrm{~V}_{3} \mathrm{M}_{2}$ recorded highest amount of nutrient uptake by rice. Further it is to be mentioned that Oppm As and flood condition had better influence on the nutrient content and uptake by rice. For further confirmation field trial is needed.

\section{Acknowledgements}

The authors are grateful to Ministry of National Science and Technology (NST), Bangladesh and Bangladesh Institute of Nuclear Agriculture (BINA) for providing financial and technical support to carry out this work.

\section{References}

Begum, M., Akter, J., Jahiruddin, M. and Islam, M.R. 2008. Effects of arsenic and its interaction with phosphorous on yield and arsenic accumulation in rice. J. Bangladesh. Agril. Univ. 6 (2): 277-284.

Horswell, J., Speir, T. 2006. Arsenic phytotoxicity: Effect on crop yield and crop quality. In: Managing Arsenic in the Environment: From Soil to Human (CSIRO). Melbourne, Australia. 183-207.

Islam, M.R. and Jahiruddin, M. 2010. Effects of arsenic and its interaction with phosphorus on yield and arsenic accumulation in rice. $19^{\text {th }}$ World Congress of Soil Science, Soil Solution for a Changing World, Brisbane, Australia.

Marin, A.R., Masscheleyen, P.H. and Patrick, W.H.J. 1992. The influence of chemical form and concentration of arsenic on rice growth and tissue arenic concentration of arsenic on rice growth and tissue arsenic concentration. Plant Soil. 139: 175183.

Meharg, A.A. and Rahman, M.M. 2003. Arsenic contamination of Bangladesh paddy field soils: implication for rice contribution to arsenic consumption. Environ. Sci. Technol. 37(2): 229-234.

Rahman, M.A., Hasegawa, H., Rahman, M.M., Rahman, M.A. and Miah, M.A.M. 2007. Accumulation of arsenic in tissues of rice plant (Oryza stiva L.) and its Distribution in fractions of rice grain. Chemosphere. 69(6): 942-948.

Rahman, M.A., Hasegawa, H., Rahman, M.M., Miah, M.A.M. and Tasmin, A. 2008. Straighthead disease of rice (Oryza sativa L.) induced by arsenic toxicity. Environ. Experiment Bot. 62(1): 54-59.

Wang, F.M., Chen, Z.L., Zhang, L., Gao, Y.L. and Sun, Y.X. 2006. Arsenic uptake and accumulation in rice (Oryza sativa L.) at different growth stages following soil incorporation of roxarsone and arsanilic acid. Plant Soil. 285(1\&2): 359-367.

Xie, Z.M. and Huang, C.Y. 1998. Control of arsenic toxicity in rice plants grown on an arsenic-polluted paddy soil. Commun. Soil Sci. Plant Anal. 29: 2471-2477.

Zhu , Y.G. 2003. Do phosphorus nutrition and iron plaque alter arsenate (As) uptake by rice seedlings in hydroponic culture? New Phytologist (2003) 162: 481-488. 